\title{
Development of a System for In Vitro Neck Muscle Force Replication in Whole Cervical Spine Experiments
}

\author{
Manohar M. Panjabi, PhD, ${ }^{*}$ Takehiko Miura, MD, ${ }^{*}+$ Peter A. Cripton, PhD, ${ }^{*}$ \\ Jaw-Lin Wang, PhD, ${ }^{*} \ddagger$ Amrinder S. Nain, MS, ${ }^{*}$ and Christian DuBois, MD*
}

Study Design. An in vitro biomechanical study.

Objectives. To develop and evaluate a new in vitro whole cervical spine model that provides to the specimen, in vivo-like mechanical characteristics.

Summary of Background Data. In vitro studies of kinematics, kinetics, and trauma using isolated spine specimens (head-T1 vertebra) have usually applied upward force to the head, resulting in tensile spine forces, contrary to the physiological compressive forces present in vivo. Further, the in vitro load-displacement curves have never been compared with the corresponding in vivo data.

Methods. A novel muscle force replication (MFR) system is presented. It consists of a set of compressive forces applied to the various vertebrae and occiput of a whole cervical spine specimen. Two protocols, with and without MFR, were evaluated using standardized flexibility testing. Ranges of motion (ROM) and load-displacement curves were documented, and contrasted with similar in vivo data.

Results. Results for the MFR were found to be similar to the in vivo measurements, with respect to the intersegmental and whole neck motions as well as the load-displacement curves, thus validating the MFR approach.

Conclusions. The new model advances the in vitro testing, which uses whole cervical spine specimens. Key Words: in vitro models, whole cervical spine, axial preload, biomechanics] Spine 2001;26:2214-2219

In vitro biomechanical studies are useful for documenting basic biomechanical functions of the spine, ${ }^{15}$ variations caused by age, ${ }^{4}$ degeneration, ${ }^{12}$ injuries caused by trauma, ${ }^{18}$ and evaluation of fixation devices. ${ }^{7}$ They may also be used to simulate in vivo situations, such as whiplash trauma. Every model (physical, in vitro, in vivo, and computer), ${ }^{21}$ provides a certain aspect of the in vivo reality. In vitro models, especially those using human cadaveric spine specimens, have certain advantages. The in vitro model has anatomy, physical properties of the soft tissues, and mechanical properties of the joints that are similar to those of the human. The in vitro model has the possibility to allow the application of known force and moment vectors, to have higher accuracy of motion mea-

From the *Biomechanics Research Laboratory, Department of Orthopaedics and Rehabilitation, Yale University School of Medicine, New Haven, Connecticut, the †Department of Orthopaedic Surgery, St. Marianna University School of Medicine, Kanagawa, Japan, and the $\ddagger$ Institute of Biomedical Engineering, National Taiwan University, Taipei, Taiwan.

Acknowledgment date: December 22, 2000.

First revision date: March 15, 2001.

Acceptance date: March 19, 2001.

Device status category: 1 .

Conflict of interest category: 12 . surement, to document three-dimensional intersegmental spinal motions including coupled motions, to have in vivo-like load displacement curves, and has the possibility to simulate spinal loading conditions that are more like those seen in vivo. Some of these potential advantages have yet to be realized.

To perform an in vitro biomechanical study, the spine specimen is provided with end fixations, often in the form of quick setting epoxy mounts, which incorporate parts of the end vertebrae. Additionally, the mounts provide fixation to the test table and to the loading fixtures. The weight of the upper mount, and also the weight of the head or head surrogate if present, must be carried so as not to buckle the specimen. It is known that a whole cervical spine specimen $(\mathrm{C} 0-\mathrm{T} 1)$ buckles under loads greater than $11 \mathrm{~N},{ }^{17}$ whereas the in vivo load has been estimated to range between 53 and $1175 \mathrm{~N}$ depending upon the posture. ${ }^{6,12}$ The muscles in vivo help generate these large compressive spinal loads and also stabilize the spine. These muscle effects are lacking in the in vitro studies. In a generic in vitro setup, an upward force is applied to the upper mount to balance the weight of the top mount and head (Figure 1). The spine specimen is subjected to near zero or some tensile force, which is nonphysiological. Using the concept of follower force (Figure 2) Patwardhan and coworkers developed a novel method of applying physiological compressive force throughout the spine specimen. ${ }^{22,23}$

There have been several in vitro studies of the kinematics of the cervical spine in which the motions of the spine have been documented for each intervertebral level from $\mathrm{C} 0-\mathrm{C} 1$ to $\mathrm{C} 7-\mathrm{T} 1$. Similarly, there are in vivo studies in which ranges of motion for the whole and intersegmental levels, especially in flexion/extension, have been recorded. In general, there is reasonable agreement between the ranges of motion of the in vitro and in vivo studies; especially considering the variability of the patients, age groups, and the methodologies used in the two study types. However, to our knowledge, there are no in vitro studies that have attempted to obtain the loaddisplacement curves similar to those measured in vivo. The load-displacement curve documents the rotation of a segment as a function of the applied moment in a continuous manner. It therefore provides a more complete description of the mechanical characteristics of a joint than does the range of motion.

The authors of the present study hypothesize that an in vitro cervical spine model can be developed that will exhibit the in vivo-like behavior with respect to the in- 


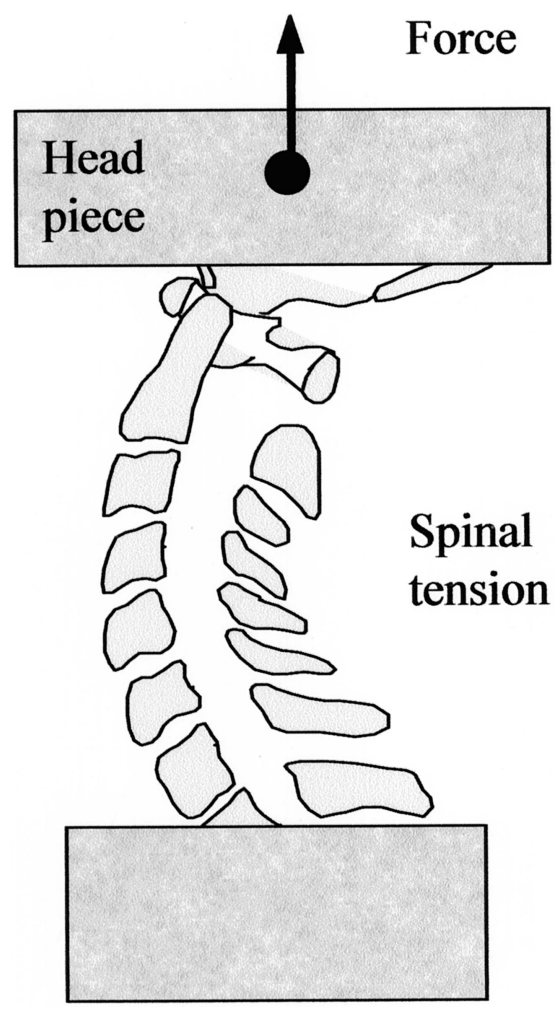

Figure 1. In vitro experiments using spine specimens require stabilizing forces, especially when carrying a head-piece. An upward force is applied at the center of gravity of the head to counteract the weight of the head-piece, which may include upper mount, loading fixture, and head surrogate.

tersegmental ranges of motion, spinal loads, and loaddisplacement curves. Thus, the purpose of the study was to develop such a model replicating the actions of the muscle forces, and to perform an experiment to validate its behavior against the in vivo data.

\section{- Materials And Methods}

Specimen Preparation. Six human cervical spines were harvested from donors (average age 44 years, range $28-59$, all male) within 24 hours of death and frozen immediately. The donors had not suffered head or neck trauma, nor had they suffered from any disease known to affect spinal biomechanics (e.g., rheumatoid arthritis or osteoporosis). Caudally, the spines were transected inferior to T1. To include the craniocervical structures, the occipital bone was transected $5 \mathrm{~cm}$ above the foramen magnum along the transverse plane. Thus the specimen consisted of the occiput and eight vertebrae. The specimen was wrapped with saline-soaked gauze, packed in double plastic bags, and stored frozen at $-20^{\circ} \mathrm{C}$.

Before testing, the specimen was thawed and screened radiographically for abnormal degeneration or other pathologies. Muscular tissue was carefully dissected from the specimen. At all stages of preparation, care was taken not to injure or remove ligaments, joint capsules, disc annulus or parts of the vertebrae. C0 (the occiput) and T1 vertebra were set into epoxy blocks, which were horizontal and parallel to each other. The specimen was oriented so that the foramen magnum was horizontal and the C6 vertebra was tilted in $20^{\circ}$ of flexion with respect to the horizontal plane, simulating normal spine neutral posture. ${ }^{2}$ The epoxy blocks were subsequently used to attach the specimen to the test table and loading apparatus.

The MFR System. The set of compressive forces designed to simulate the load carrying and stiffening functions of the neck muscles was termed the muscle force replication (MFR) system. There were lateral (left-right) and anterior-posterior forces applied to each vertebra, constituting the MFR system. The MFR forces were applied via $0.6-\mathrm{mm}$ monofilament nylon cables. This cable had the advantage of low friction and it was hydrotropic. To serve as guides for the MFR cables, 3.0-mm diameter stainless steel rods were inserted laterally through each vertebral body and through the $\mathrm{C} 0$ block (Figure 3). The rods were inserted under fluoroscopic control to prevent damage to the endplates or other structures and to confirm a laterally directed path. The guides were inserted at approximately the location of the flexion-extension centers of rotation (COR). ${ }^{1,5,27}$ Because the $\mathrm{C} 1$ vertebra has no vertebral body, no rod was inserted at this level.

Small self-tapping eyelets were inserted into the vertebral bodies from anterior and used as guides for the anterior MFR cables. Posteriorly, MFR guidance was accomplished by tying wire loops through holes drilled in the spinous processes.

Lateral MFR Forces. Laterally, MFR cables were anchored at guide rods passing through the $\mathrm{C} 0$ block which was approximately at the $\mathrm{COR}$ of $\mathrm{C} 0-\mathrm{C} 1$, and $\mathrm{C} 2, \mathrm{C} 4$, and $\mathrm{C} 6$ vertebrae (Figure 3). As the cables ran down the spine, they passed alternately anterior and posterior to the guide rods. All lateral MFR forces were applied bilaterally. Each MFR cable passed through pulleys at the T1 block of the specimen.

A tension of $30 \mathrm{~N}$ was applied to each cable by hanging weights from the cable ends. Moving caudally, the segmental compression forces thus increased with each second vertebra to a maximum of $240 \mathrm{~N}$ at C7 (Table 1). This is consistent with the known in vivo loads in the cervical spine, which have been

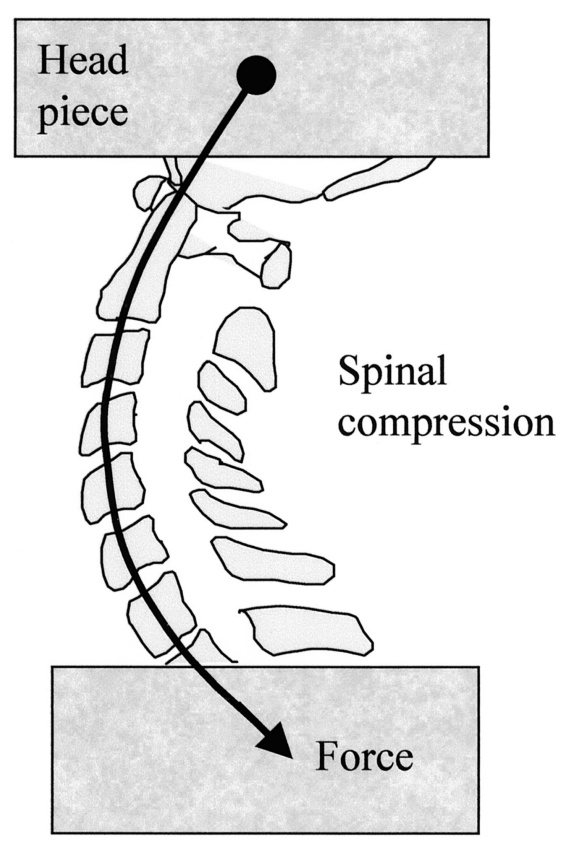

Figure 2. Recently, it has been shown that a follower compressive force passing through the centers of rotation of each spinal segment can apply large physiological compressive forces to the spine. 


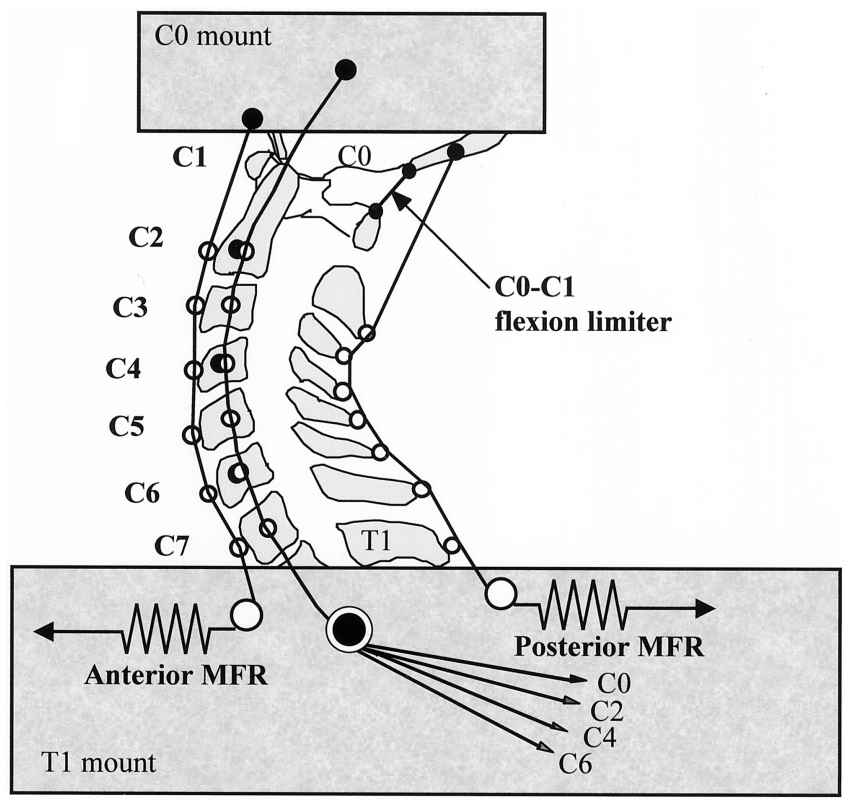

Figure 3. The muscle force replication (MFR) system. For the Lateral MFR, the cables are anchored at C0, C2, C4 and C6, and are guided to pass through intersegmental centers of rotation. For the anterior and posterior MFR, both cables are anchored at the $\mathrm{CO}$, and are guided to pass on the anterior and posterior aspects of each vertebra, respectively.

estimated to range between 53 and $1175 \mathrm{~N}$ depending upon the posture. $^{6,11}$

The naturally high mobility at the craniocervical junction $(\mathrm{C} 0-\mathrm{C} 2)$ and the lack of guide rods at C1 complicated the selection of an anchor point for the lateral MFR cables. An anterior anchor location resulted in the C0-C1-C2 levels becoming locked in flexion irrespective of the external moment applied. Similarly, a posterior location resulted in an extensionlocked posture at the upper cervical spine. Therefore, a device was constructed to allow fine anterior-posterior adjustment of the lateral MFR cable anchor point on C0 (Figure 3). Using this device, a point could be identified at which the application of compressive force to the lateral cables resulted in neither flexion nor extension rotation of $\mathrm{C} 0$ or $\mathrm{C} 1$ with respect to $\mathrm{C} 2$. This phenomenon has previously been reported in the lumbar spine for single segments. ${ }^{19,26,28}$ The point of application is sometimes referred to as the balance point of the segment.

Anterior-Posterior MFR Forces. The anterior and posterior MFR cables were anchored to the occiput and ran through the eyelets anteriorly and through the wire loops posteriorly (Figure 3). The cables ran through pulleys at the T1 block. They were then each attached to separate springs. The springs had a stiffness coefficient of $1.0 \mathrm{~N} / \mathrm{mm}$ and were adjusted to have no pre-tension with the specimen in the neutral posture. No gap was allowed at the spring connection, thus force was produced immediately in the anterior and posterior MFR cables with any extension or flexion of the specimen.

C0-C1 Flexion Limiter. The osteoligamentous cervical spine allows a non-physiologic hyperflexion at the C0-C1 level. Anatomic elements that prevent the hyperflexion in vivo probably include chin contact with the sternum anteriorly, and active and passive resistance of the posterior muscles. To mimic this effect, a flexion limiter was developed which allowed only physiologic $\mathrm{C} 0-\mathrm{C} 1$ flexion. A wire was tied between the posterior arch of $\mathrm{C} 1$ and the posterior margin of the occiput (Figure 3$)$. The wire length was adjusted such that the flexion rotation under $3.6 \mathrm{Nm}$ flexion moment was limited to $15^{\circ} \pm 1^{\circ}$. This value is consistent with the in vivo flexion for this level reported by Ordway et al. ${ }^{14}$

The Flexibility Tests. To determine the intervertebral motions in response to the applied pure moments in the sagittal plane, previously developed methodology ${ }^{20}$ was adopted to the present study.
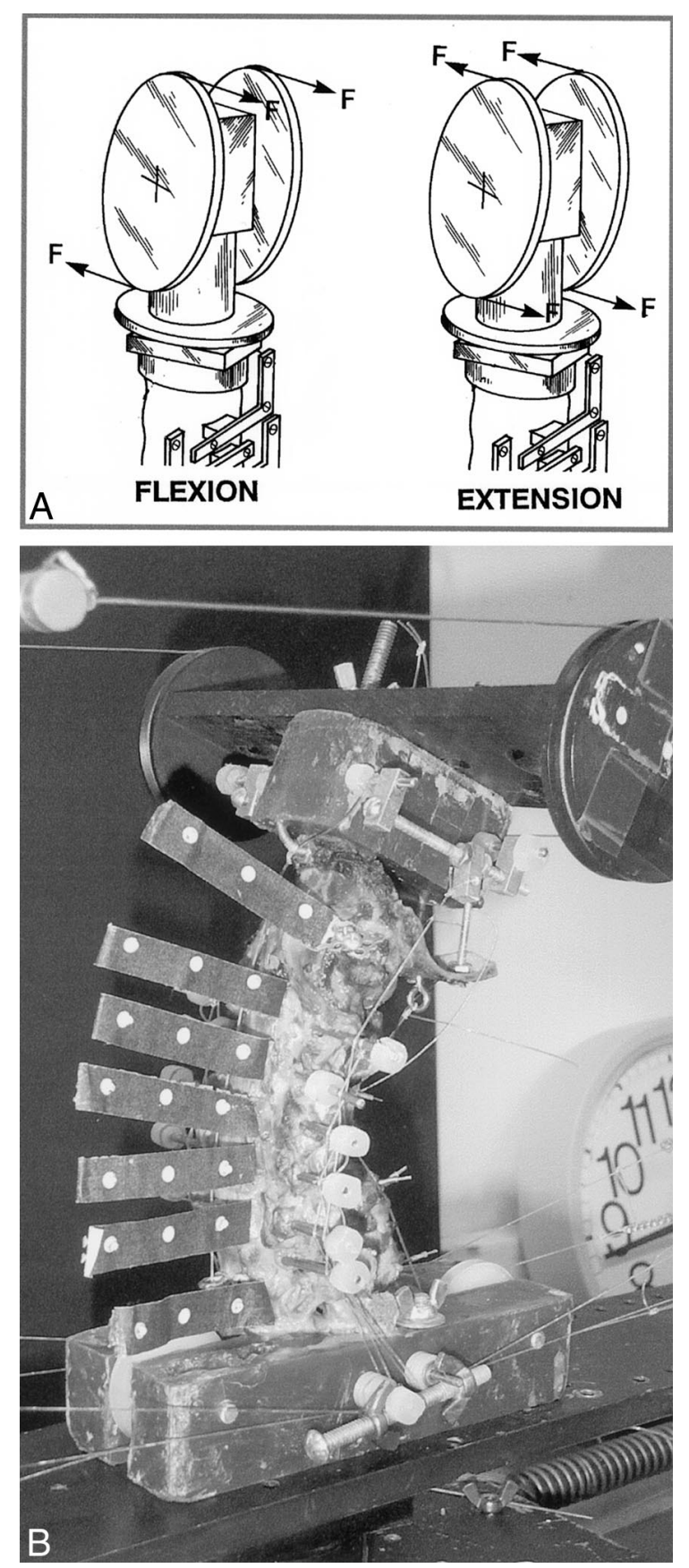

Figure 4. Flexibility testing of the specimen with MFR. A, Schemetics showing the flexibility set up, where the specimen is loaded with pure flexion and extension bending moment during flexibility testing. B, Photograph of the specimen with the MFR system. 


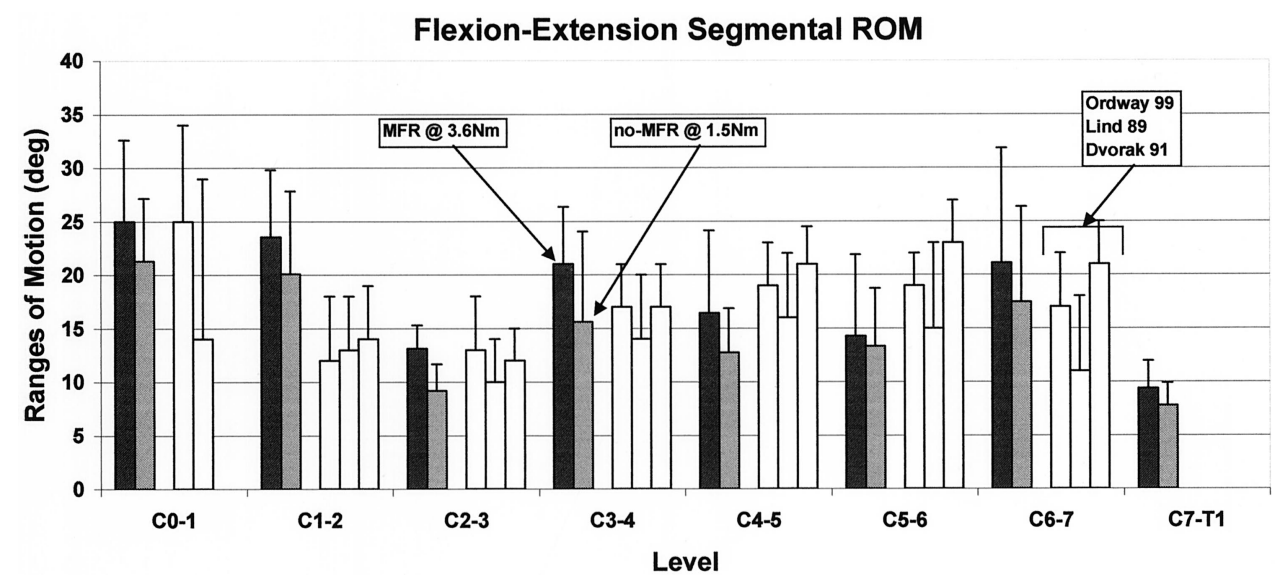

Figure 5. Average (one standard deviation) total ROMs (flexion plus extension at $3.6 \mathrm{Nm}$ ) for all levels in the presence of MFR are shown. Also included are the corresponding results of three in vivo studies for comparison.

Moment Application. Pure flexion and extension moments were applied to the $\mathrm{C} 0$ block using a custom-loading jig, while the T1 block was fixed to the test table (Figure 4A). The loading jig consisted of two 7-cm diameter pulleys attached bilaterally to the C0 mould. Equal and opposite forces were applied to the pulley rims using wire and hanging weights. This resulted in pure moments (i.e., no compression or shear forces) being applied to the specimen. The height of the wires was adjusted during the loading to ensure that the applied moment remained pure and the specimen moved naturally without constraint. The moments were applied in four equal steps.

Kinematics Measurements. Plane sagittal rotations of each vertebra and the occiput were measured using a digital motion analysis camera (motion analyzer model PS-110, Eastman Kodak Company, Rochester, NY, USA), and custom software. Each vertebra and the occiput had a black motion flag attached anteriorly with three white targets (Figures 4B). A digital image was collected at each moment step. The coordinates of the centroids of the targets were digitized. To maximize measurement accuracy, targets on each flag, which were closest and farthest from the specimen, were used to determine the global and intervertebral rotations. An experiment was performed to determine the repeatability, which includes both the errors of the measurement system and the reproducibility of the biomechanical response to the applied loads. Performing three flexibility tests separated by 2 hours of rest between segmental tests, the standard deviation of the intervertebral motion differences was 0.7 degrees.

Experimental Protocols. Each specimen was tested in two protocols: traditional (i.e., no MFR) and the new (with MFR). In the no-MFR protocol, a vertical upward force was applied to the $\mathrm{C} 0$ mount to counteract the weight of the mount and the loading jig (Figure 1). Maximum moment applied was $1.5 \mathrm{Nm}$, achieved, starting from zero, in four steps, 0.375, 0.75, 1.125, and $1.5 \mathrm{Nm}$. With MFR protocol, the maximum moment was 3.6 $\mathrm{Nm}$ reached in steps: $0.9,1.8,2.7$, and $3.6 \mathrm{Nm}$, starting from zero. At each moment step, the loading was held constant for 30 seconds to allow for viscoelastic creep. In both flexion and extension, two so-called preconditioning cycles ${ }^{3,24}$ were applied but no data were collected. Thereafter, a third loading cycle was applied from which the kinematics data were recorded.

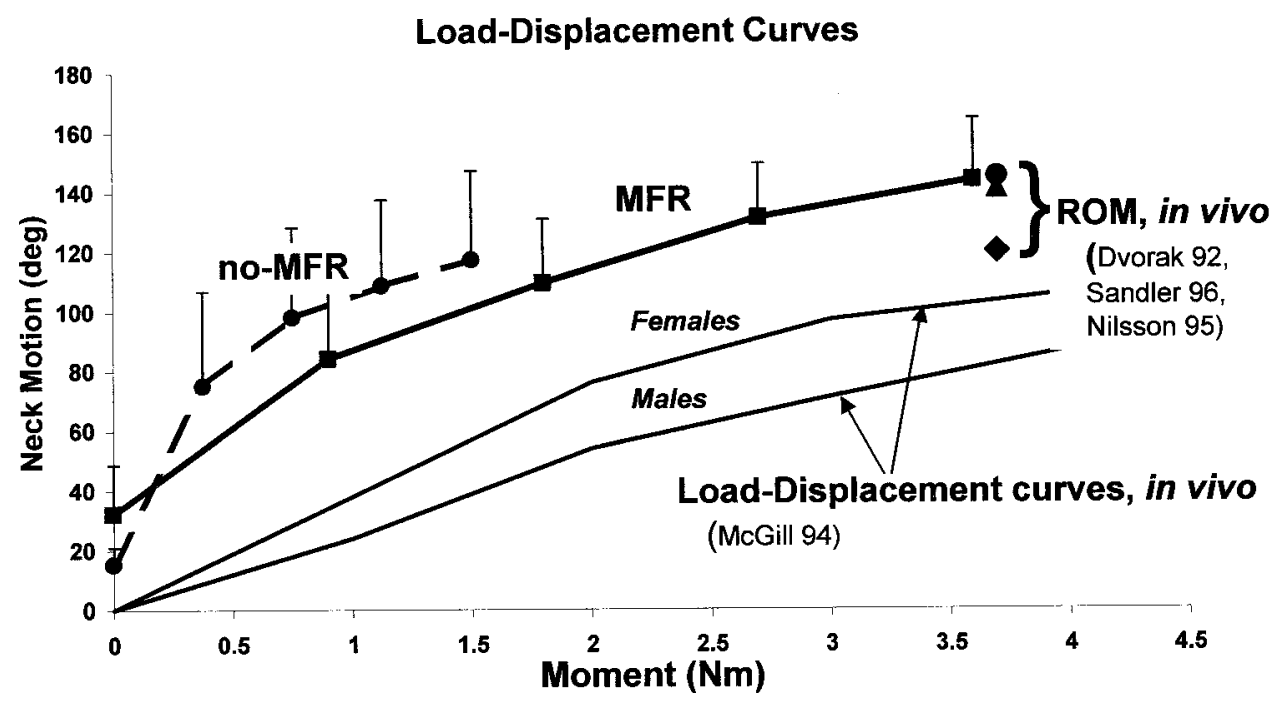

Figure 6. Average (one standard deviation) moment-rotation curves of the specimens without MFR and with MFR are shown. Included are total ROM values from three in vivo studies for comparison. 


\section{nesults}

\section{Intervertebral Kinematics}

The segmental flexion-extension ranges of motion (ROM, equal to the sum of flexion and extension angles measured at maximum moment) were compared to the in vivo data from several in vivo sources ${ }^{5,8,14}$ (Figure 5). The highest ROMs occurred at $\mathrm{C} 0-\mathrm{C} 1, \mathrm{C} 1-\mathrm{C} 2$ and C6-C7 levels (approximately 20 to $25^{\circ}$ ). The lowest ROMs were measured at C2-C3 (approximately $13^{\circ}$ ). The results were in excellent overall agreement with the in vivo data. There were no significant differences between the no-MFR and MFR results.

\section{Load-Displacement Curves}

The load-displacement or moment-angle curves for both no-MFR and MFR were nonlinear, showing stiffening with increasing load (Figure 6). For in vivo comparison, two sets of data were available, which are also shown in the figure. The first is the range of motion data. ${ }^{4,13,25}$ Both the no-MFR and MFR have in vivo-like ranges of motion. The second data set is the moment-angle curve. ${ }^{10}$ The MFR curve is similar to the in vivo curve, but the no-MFR curve is not.

\section{Discussion}

The spine specimen devoid of musculature is incapable of carrying the weights of the mount, loading jig and head or head surrogate. Researchers routinely apply an upward force to the center of gravity of the combined weight on top of the specimen, ${ }^{9,16,20}$ so as to carry the weight and prevent buckling of the specimen. This method results in either zero load or some tension in the spine specimen. This load is not physiological, as the in vivo spine carries significant compressive load.

The study has shown that a spine specimen with MFR system can carry the weight of the head and mimic the in vivo intersegmental motions, whole neck motion, and stiffness of the neck. The results show excellent agreement with the in vivo measurements. When it comes to the ranges of motion of the whole neck and each intervertebral segment, the traditional in vitro methodology has produced results that are in excellent agreement with the in vivo data, as illustrated in Figure 5. But no study has produced the in vitro load-displacement or moment-angle curves similar to those measured in vivo. The no-MFR curve of the present study clearly shows the lack of agreement between the in vitro and in vivo results (Figure 6). The MFR curve, on the other hand, shows remarkable likeness in shape to the in vivo data, but is shifted upward on the motion axis. The authors of the present study believe that the lack of exact match between the MFR and in vivo curves is caused by the unnaturally high stiffness of the in vivo data. In the in vivo study, the patients lie on a horizontal platform on their sides with frictionless support under the head. When the moment was applied to the head to produce motion, the patients were instructed to relax their neck muscles. Because of the testing arrangement, it is conceivable that the patients were not fully relaxed at the time of testing. In that situation, their range of motion will be expected to be less than the normal in vivo data. We find support for this argument when we compare the ranges of motion of the two in vivo study types (Figure 6). The load-displacement study ${ }^{10}$ has a range of motion considerably smaller than the average range of motion of the other three in vivo studies. ${ }^{4,13,25}$ On the other hand, the range of motion of the present study is nearly identical to that of the three in vivo studies.

Presently, we lack full understanding of the mechanics involved with the MFR system. It is easy to see that the forces applied to the left and right side of the specimen provide bending moment in the frontal plane in response to an external disturbing moment in that plane. This helps stabilize the specimen. The resisting sagittal plane moment is provided by the tension developed in the anterior and posterior springs of the MFR system, as the spine flexes and extends (Figure 3). However, because of altered properties of the disc and facet interactions by the MFR forces, the contributions are presently unknown. Future research may address this important aspect of the MFR system.

A limitation of the present study is the uncertainty of the location of the guide rods that are placed laterally into each vertebral body. Ideally, the rods should be placed at the respective centers of rotation of intervertebral motions. However, the centers of rotation may change during the flexion/extension motion. Thus, the placement of the guide rods is approximate. The vertebral bodies are violated by the insertion of the guide rods. This excludes the use of the MFR model, at least in the present configuration, for studies that need intact vertebral bodies (e.g., spinal instrumentation studies). A different approach using preloads has been suggested, which does not violate the vertebral bodies and is therefore better suited to the study of instrumentation testing, which needs intact vertebral bodies. ${ }^{11}$

\section{Conclusions}

The authors of the present study have developed a new methodology to support the weight of the head and stabilize the osteoligamentous specimen, while using whole cervical spine specimens in in vitro experiments. The methodology, termed MFR, includes the application of a set of compressive muscle replication forces to various vertebrae. Based upon the experimental studies, the following conclusions can be drawn:

With MFR the specimen is subjected to physiological compressive force, which improves the model's biofidelity.

With MFR the specimen exhibited kinematic characteristics, at intervertebral levels and of the whole cervical spine, which were similar to the in vivo measurements. 
With MFR the in vitro load-displacement curve for the whole cervical spine obtained was similar to the in vivo curve.

\section{- Key Points}

- The new muscle force replication (MFR) system advances the in vitro testing methods, so that a whole cervical spine specimen in the laboratory may behave more like an in vivo subject.

- The MFR system provides in vivo-like spinal loads.

- The MFR system provides in vivo-like whole cervical spine load-displacement behavior.

- The MFR system provides in vivo-like whole cervical spine and segmental ranges of motion.

\section{Acknowledgements}

This research was supported in part by Volvo Research Foundation, Volvo Educational Foundation, Dr. Pehr Gyllenhammar Research Foundation, National Institutes of Health, and Natural Sciences \& Engineering Research Council of Canada.

\section{References}

1. Amevo B, Worth D, Bogduk N. Instantaneous axes of rotation of the typical cervical motion segments: a study in normal volunteers. Clinical Biomechanics 1991;6:111-7.

2. Braakman R, Penning L. Injuries of the cervical spine. The Netherlands: Excerpta Medica, 1971.

3. Crisco JJ, Oda T, Panjabi MM, et al. Transections of the C1-C2 joint capsular ligaments in the cadaveric spine. Spine 1991;16(Suppl):S474-9.

4. Dvorak J, Antinnes JA, Panjabi MM, et al. Age and gender related normal motion of the cervical spine. Spine 1992;17(Suppl):S393-8.

5. Dvorak J, Panjabi MM, Novotny JE, et al. In vivo flexion/extension of the normal cervical spine. Journal of Orthopaedic Research 1991;9:828-34.

6. Hattori S, Oda H, Kawai S, et al. Cervical intradiscal pressure in movements and traction of the cervical spine. Z Orthop 1981;119:568-9.

7. Isomi T, Panjabi MM, Wang JL, et al. Stabilizing potential of anterior cervical plates in multi-level corpectomies. Spine 1999;24:2219-23.

8. Lind B, Sihlbom H, Nordwall A, et al. Normal range of motion of the cervical spine. Arch Phys Med Rehabil 1989;70:692-5.

9. Lund T, Oxland TR, Jost B, et al. Interbody cage stabilisation in the lumbar spine: biomechanical evaluation of cage design, posterior instrumentation and bone density. J Bone Joint Surg [Br] 1998;80:351-9.

10. McGill SM, Jones K, Bennett G, et al. Passive stiffness of the human neck in flexion, extension, and lateral bending. Clin Biomech 1994;9:193-8.

11. Miura T, Panjabi MM, Cripton PA. A method to simulate in vivo cervical spine kinematics using in vitro compressive preload. Spine 2001, in press.
12. Moroney SP, Schultz AB, Miller JA. Analysis and measurement of neck loads. J Orthop Res 1988;6:713-20.

13. Nilsson N. Measuring passive cervical motion: a study of reliability. J. Manipulative Physiol Ther 1995;18:293-7.

14. Ordway NR, Seymour RJ, Donelson RG, et al. Cervical flexion, extension, protrusion, and retraction. A radiographic segmental analysis. Spine 1999; 24:240-7.

15. Panjabi MM, Crisco JJ, Vasavada A, et al. Three-dimensional physical properties of the cervical spine as shown by the load-displacement curves. Spine, in press.

16. Panjabi MM, Cholewicki J, Nibu K, et al. Simulation of whiplash trauma using whole cervical spine specimens. Spine 1998;23:17-24.

17. Panjabi MM, Cholewicki J, Nibu K, et al. Critical load of the human cervical spine: an in vitro experimental study. Critical Load Clin Biomech 1998;13: $11-7$.

18. Panjabi MM, Cholewicki J, Nibu K, et al. Mechanism of whiplash injury. Clinical Biomechanics 1999;13:239-49.

19. Panjabi MM, Krag MH, White AA. Effects of preload on load-displacement curves of the lumbar spine. Orthop Clin North Am 1977;8:181-91.

20. Panjabi MM, Oxland TR, Lin RM, et al. Thoracolumbar burst fracture: a biomechanical investigation of its multidirectional flexibility. Spine 1994;19: $578-85$.

21. Panjabi MM. Cervical spine models for biomechanical research. Spine 1998; 23:2684-700.

22. Patwardhan AG, Havey RM, Ghanayem AJ, et al. Load-carrying capacity of the human cervical spine in compression is increased under a follower load. Spine 2000;25:1548-54.

23. Patwardhan AG, Havey RM, Meade KP, et al. A follower load increases the load-carrying capacity of the lumbar spine in compression. Spine 1999;24: 1003-9.

24. Pelker RR, Duranceau JS, Panjabi MM. Cervical spine stabilization. A threedimensional, biomechanical evaluation of rotational stability, strength, and failure mechanisms. Spine 1991;16:117-22.

25. Sandler AJ, Dvorak J, Humke T, et al. The effectiveness of various cervical orthoses: an in vivo comparison of the mechanical stability provided by several widely used models. Spine 1996;21:1624-9.

26. Tencer AF, Ahmed AM. The role of secondary variables in the measurement of the mechanical properties of the lumbar intervertebral joint. Journal of Biomechanical Engineering 1981;103:129-37.

27. van Mameren H, Sanches H, Beursgens J, et al. Cervical spine motion in the sagittal plane. II. Position of segmental averaged instantaneous centers of rotation-a cineradiographic study. Spine 1992;17:467-74.

28. Wilder DG, Pope MH, Seroussi RE, et al. The balance point of the intervertebral motion segment: an experimental study. Bulletin/Hospital for Joint Diseases 1989;49:155-69.

\section{Address reprint requests to}

Manohar M. Panjabi, PhD Professor \& Director

Biomechanics Laboratory

Department of Orthopaedics and Rehabilitation Yale University School of Medicine P.O. Box 208071

New Haven, CT 06520-8071 E-mail:manohar.panjabi@yale.edu 